\title{
EDITORIAL
}

\section{Systems Biology: A Primer}

In this issue of NeuroRx ${ }^{\circledR}$ we present a collection of articles that illustrate the application of methodologies in systems biology for advancing our understanding of the nervous system, its disorders, and potential therapies. But what is "systems biology?" Some may define this term in a historical manner harkening back to systems neuroscientists who have laid the foundations for the anatomical and electrophysiological workings of the brain. Others may be drawn to a more clinical, perhaps broader definition focused on the functions and dysfunctions of a particular organ system (e.g., the cardiovascular system). However, while both have utility, they remain self-limiting definitions, constrained by their particular historical or anatomical derivations.

Over the past decade the term "systems biology" has evolved into a more encompassing, perhaps even "holistic," term. Leroy Hood, president and cofounder of the Institute for Systems Biology (ISB) in Seattle, has described systems biology succinctly as "studying the interactions and interplay of many levels of biological information," which will lead toward the "new era of predictive, preventive, and personalized medicine" (www.systemsbiology.org). Taken to the extreme, systems biology will entail understanding the workings of all biological molecules on all levels, from the subcellular organelle to the complete organism. By necessity and, more importantly, by design, systems biology investigation requires interdisciplinary collaboration, from basic scientists to clinicians and from biostatisticians to computer scientists. In essence it transcends the traditional "boundaries" of scientific and medical study. And no other field seems better primed for study on this level than understanding the nervous system, its disorders, and potential neurotherapeutics.

While no single issue nor series of issues could hope to fully convey the burgeoning field of systems biology, there is a need to begin to frame and conceptualize the study of nervous system disorders and therapies along these lines. Systems biology in general and its application to the nervous system in particular remain in their infancy. However, a number of recent advances in highthroughput "-omics" technologies have allowed both basic scientists and clinicians alike the opportunity to scan

Address correspondence and reprint requests to: Howard J. Federoff, M.D., Ph.D., Department of Neurology, Center for Aging and Developmental Biology, Box 645, University of Rochester School of Medicine and Dentistry, 601 Elmwood Avenue, Rochester, NY 14642. the various genomes, transcriptomes, proteomes, and metabolomes of human tissues and animal models of disease. Furthermore, strides in computer and statistical modeling are beginning to allow new insights into the high-dimensional data generated from these studies. Thus, we have intentionally designed this issue to be a primer to systems biology, a set of illustrations that any researcher, regardless of area of expertise, can appreciate and understand as this exciting new field will surely revolutionize the field of neurotherapeutics. As such, we have taken a didactic approach and provided articles by experts that progress from genes to transcripts, proteins to metabolites, and finally to methods of analysis-all in relation to neurological disorders and potential insights into future therapies.

The issue's first article deals with an aspect of genetics of neurological disorders and treatments. A review by Uhl focuses on identifying and understanding potential addiction vulnerability genes and how these may be used to manage the risk of dependence on a number of neurotherapeutics that have the potential for abuse, including drugs used to treat pain, seizures, and other disorders.

The next set of reviews focuses on the study of gene transcripts in neurological disorders and their potential application to therapeutic development. Ginsberg and colleagues discuss studies of gene expression on the single cell level and how these may inform drug development in mild cognitive impairment and Alzheimer's disease (AD). Miller and Federoff review microarray studies in human Parkinson's disease and animal models to illustrate the potential to identify susceptibility markers, disease biomarkers, and therapeutic targets.

These are followed by a set of three reviews that discuss proteomic technologies and their applications to neurological disorders. Choe and coworkers review twodimensional protein electrophoresis methods and studies that are beginning to elucidate molecular pathways of neurological disease and illustrate their potential for the discovery and validation of disease and therapeutic biomarkers. Next, Montine and colleagues discuss the use of liquid chromatography-electrospray ionization-tandem mass spectrometry-based proteomics to understand proteomic changes in both the aging human brain and in neurological disorders, including AD. Butterfield and coworkers then review a specific subset of proteomics, redox proteomics, and describe its application to studies in a number of neurodegenerative disorders. 
In the final review of "-omics" technologies, Holmes and coworkers review metabolomic/metabonomic technologies and their use in metabolic profiling in neurodegenerative diseases, disease progression, and therapeutic interventions.

The issue's last three reviews are oriented toward methodologies in the analysis of biological data. Olson reviews the importance of experimental design, data accessibility, platform selection, statistical analysis, and determination of biological significance in microarray studies. In a bit of a departure from a strictly nervous system focus, Almudevar and coworkers discuss the use of correlation measures in analyzing microarray data and in reconstructing gene regulatory networks in human disease. While the authors illustrate their points using data from the cancer field, they discuss the applicability of these statistical methodologies to the study of nervous system disorders and potential therapeutics. Finally, Ma'ayan and coworkers bring this issue full circle by reviewing the use of network analysis, coupled with future testing in animal models, to understand Down syndrome and how these applications may lead to future therapies. In other words they begin to illustrate the full potential of systems biology in understanding neurological disorders and its application to neurotherapeutics.
Thus, these ten reviews lay the conceptual framework of the future of systems biology in neurotherapeutics research. Based upon the technologies, methodologies, and science presented by these authors, coupled with the work of countless basic scientists, clinicians, computer scientists, and bioinformaticists and biostatisticians worldwide, we predict an explosion of systems biologybased research in the coming years. Importantly, we hope that the reader will see the utility of these reviews, especially in relation to the past and future issues of NeuroRx $x^{\circledR}$. It is at this point when one truly sees the importance of the cross-collaborative disciplinary efforts of systems biology; the potential to combine multilevel information on biological molecules with clinical and imaging data to inform our understanding of neurological disorders and their diagnosis, progression, and treatments. It is at this point that, to paraphrase Dr. Hood, we will truly begin the "new era of predictive, preventive, and personalized medicine."

Howard J. Federoff, M.D., Ph.D. E-mail: howard_federoff@urmc.rochester.edu Timothy R. Mhyre, Ph.D. E-mail: timothy_mhyre@urmc.rochester.edu Guest Editors 